\title{
SOME 3-ADIC CONGRUENCES FOR BINOMIAL SUMS
}

\author{
YONG ZHANG AND HAO PAN
}

\begin{abstract}
We prove some 3-adic congruences for binomial sums, which were conjectured by Sun.
\end{abstract}

\section{INTRODUCTION}

For a non-zero integer $n$ and a prime $p$, let $\nu_{p}(n)$ denote the $p$-adic order of $n$, i.e., $\nu_{p}(n)$ is the largest integer such that $p^{\nu_{p}(n)} \mid n$. In [1], Strauss, Shallit and Zagier proved that for any positive integer $n$,

$$
\nu_{3}\left(\sum_{k=0}^{n-1}\left(\begin{array}{c}
2 k \\
k
\end{array}\right)\right)=2 \nu_{3}(n)+\nu_{3}\left(\left(\begin{array}{c}
2 n \\
n
\end{array}\right)\right) .
$$

Recently Sun [1] showed that

$$
\nu_{p}\left(\sum_{k=0}^{n-1} \frac{1}{m^{k}}\left(\begin{array}{c}
2 k \\
k
\end{array}\right)\right) \geq \nu_{p}(n) \quad \text { and } \quad \nu_{p}\left(\sum_{k=0}^{n-1} \frac{(-1)^{k}}{m^{k}}\left(\begin{array}{c}
2 k \\
k
\end{array}\right)\left(\begin{array}{c}
n-1 \\
k
\end{array}\right)\right) \geq \nu_{p}(n),
$$

where $m$ is an integer and $p$ is an odd prime dividing $m-4$. Furthermore, he also proposed several conjectures on the 3 -adic orders of the above two binomial sums.

Conjecture 1.1. Let $m$ be integer with $m \equiv 1(\bmod 3)$.

(i) For every positive integer $n$, we have

$$
\nu_{3}\left(\frac{1}{n} \sum_{k=0}^{n-1} \frac{\left(\begin{array}{c}
2 k \\
k
\end{array}\right)}{m^{k}}\right) \geq \min \left\{\nu_{3}(n), \nu_{3}(m-1)-1\right\} .
$$

For any integer $a \geq \nu_{3}(m-1)$, we have

$$
\frac{1}{3^{a}} \sum_{k=0}^{3^{a}-1} \frac{1}{m^{k}}\left(\begin{array}{c}
2 k \\
k
\end{array}\right) \equiv \frac{m-1}{3}\left(\bmod 3^{\nu_{3}(m-1)}\right) .
$$

(ii) For every positive integer $n$, we have

$$
\nu_{3}\left(\frac{1}{n} \sum_{k=0}^{n-1} \frac{(-1)^{k}}{m^{k}}\left(\begin{array}{c}
n-1 \\
k
\end{array}\right)\left(\begin{array}{c}
2 k \\
k
\end{array}\right)\right) \geq \min \left\{\nu_{3}(n), \nu_{3}(m-1)\right\}-1 .
$$

2010 Mathematics Subject Classification. Primary 11B65; Secondary 05A10. 
For any integer $a>\nu_{3}(m-1)$, we have

$$
\frac{1}{3^{a}} \sum_{k=0}^{3^{a}-1} \frac{(-1)^{k}}{m^{k}}\left(\begin{array}{c}
3^{a}-1 \\
k
\end{array}\right)\left(\begin{array}{c}
2 k \\
k
\end{array}\right) \equiv-\frac{m-1}{3}\left(\bmod 3^{\nu_{3}(m-1)}\right) .
$$

(iii) For any integer $a \geq 2$, we have

$$
\frac{1}{3^{a}} \sum_{k=0}^{3^{a}-1}(-1)^{k}\left(\begin{array}{c}
3^{a}-1 \\
k
\end{array}\right)\left(\begin{array}{c}
2 k \\
k
\end{array}\right) \equiv-3^{a-1}\left(\bmod 3^{a}\right) .
$$

In this paper, we shall confirm Conjecture.

Theorem 1.1. All assertions of Conjecture 1.1 are true.

The proofs of (1.3)-(1.7) will be given in the next sections.

\section{Proofs of (1.3) AND (1.4)}

For $A, B \in \mathbb{Z}$, the Lucas sequence $\left\{u_{n}(A, B)\right\}$ are given by $u_{0}(A, B)=0, \quad u_{1}(A, B)=1, \quad u_{n+1}(A, B)=A u_{n}(A, B)-B u_{n-1}(A, B)$ for $n \geq 1$. In particular, it is not difficult to check that $\left\{u_{n}(-1,1)\right\}_{n \geq 0} \in\{0,1,-1\}$ and $u_{n}(-1,1) \equiv n(\bmod 3)$.

Lemma 2.1. Suppose that $m \equiv 1(\bmod 3)$. Then we have

$$
\frac{u_{n}(m-2,1)}{n} \equiv \frac{u_{n}(-1,1)}{n}+\frac{m-1}{3}\left(\begin{array}{c}
n-1 \\
2
\end{array}\right)\left(\bmod 3^{\nu_{3}(m-1)}\right)
$$

if $m \neq 4$, and

$$
\frac{u_{n}(2,1)}{n} \equiv \frac{u_{n}(-1,1)}{n}(\bmod 3)
$$

In particular, we always have

$$
\frac{u_{n}(m-2,1)}{n} \equiv \frac{u_{n}(-1,1)}{n}\left(\bmod 3^{\nu_{3}(m-1)-1}\right) .
$$

Proof. Let $\Delta=m(m-4)$. By the properties of Lucas sequences, we have

$$
u_{n}(m-2,1)=\frac{1}{2^{n-1}} \sum_{\substack{1 \leq k \leq n \\
2 \nmid k}} \frac{n}{k}\left(\begin{array}{l}
n-1 \\
k-1
\end{array}\right)(m-2)^{n-k} \Delta^{(k-1) / 2} .
$$

If $\Delta=0$, i.e., $m=4$, then

$$
\frac{u_{n}(2,1)}{n}=\left(\frac{4-2}{2}\right)^{n-1}=1 \equiv \frac{u_{n}(-1,1)}{n}(\bmod 3) .
$$


Suppose that $\Delta \neq 0$. Then

$$
\begin{aligned}
& \frac{u_{n}(m-2,1)}{n}-\left(\frac{m-2}{2}\right)^{n-1}=\sum_{\substack{1<k \leq n \\
2 \nmid k}}\left(\begin{array}{c}
n-1 \\
k-1
\end{array}\right)\left(\frac{m-2}{2}\right)^{n-k} \frac{\Delta^{(k-1) / 2}}{k 2^{k-1}} \\
= & \sum_{\substack{1<k \leq n \\
2 \nmid k}}\left(\begin{array}{l}
n-1 \\
k-1
\end{array}\right) \frac{(m-2)^{n-k}}{2^{n-k}} \cdot \frac{((m-1)(m-3)-3)^{(k-1) / 2}}{k 2^{k-1}} \\
= & \sum_{1<k \leq n}\left(\begin{array}{l}
n-1 \\
k-1
\end{array}\right) \frac{(m-2)^{n-k}}{k 2^{n-1}} \sum_{j=0}^{(k-1) / 2}\left(\begin{array}{c}
(k-1) / 2 \\
j
\end{array}\right)(m-1)^{j}(m-3)^{j}(-3)^{(k-1) / 2-j} \\
\equiv & \sum_{\substack{1<k \leq n \\
2 \nmid k}}\left(\begin{array}{l}
n-1 \\
k-1
\end{array}\right)\left(-\frac{1}{2}\right)^{n-k} \frac{(-3)^{(k-1) / 2}}{k 2^{k-1}}+\frac{m-1}{3}\left(\begin{array}{c}
n-1 \\
2
\end{array}\right)\left(\bmod 3^{\nu_{3}(m-1)}\right) .
\end{aligned}
$$

By (2.4), it is derived that

$$
\begin{aligned}
& \sum_{\substack{1<k \leq n \\
2 \nmid k}}\left(\begin{array}{l}
n-1 \\
k-1
\end{array}\right)\left(-\frac{1}{2}\right)^{n-k} \frac{(-3)^{(k-1) / 2}}{k 2^{k-1}} \\
= & \frac{u_{n}(1-2,1)}{n}-\left(-\frac{1}{2}\right)^{n-1} \equiv \frac{u_{n}(m-2,1)}{n}-\left(\frac{m-2}{2}\right)^{n-1}\left(\bmod 3^{\nu_{3}(m-1)}\right) .
\end{aligned}
$$

We are done.

The following curious identity is due to Sun and Taurso [3, (2.1)]:

$$
m^{n-1} \sum_{k=0}^{n-1} \frac{1}{m^{k}}\left(\begin{array}{c}
2 k \\
k
\end{array}\right)=\sum_{k=0}^{n-1}\left(\begin{array}{c}
2 n \\
k
\end{array}\right) u_{n-k}(m-2,1) .
$$

It is easy to check that

$$
\frac{1}{n}\left(\begin{array}{c}
2 n \\
k
\end{array}\right)=\frac{1}{n-k}\left(2\left(\begin{array}{c}
2 n-1 \\
k
\end{array}\right)-\left(\begin{array}{c}
2 n \\
k
\end{array}\right)\right) .
$$

So (2.5) can be rewritten as

$$
\frac{m^{n-1}}{n} \sum_{k=0}^{n-1} \frac{1}{m^{k}}\left(\begin{array}{c}
2 k \\
k
\end{array}\right)=\sum_{k=0}^{n-1}\left(2\left(\begin{array}{c}
2 n-1 \\
k
\end{array}\right)-\left(\begin{array}{c}
2 n \\
k
\end{array}\right)\right) \frac{u_{n-k}(m-2,1)}{n-k} .
$$


Thus using (2.3) and (2.6), we have

$$
\begin{aligned}
\frac{m^{n-1}}{n} \sum_{k=0}^{n-1} \frac{1}{m^{k}}\left(\begin{array}{c}
2 k \\
k
\end{array}\right) & \equiv \sum_{k=0}^{n-1}\left(2\left(\begin{array}{c}
2 n-1 \\
k
\end{array}\right)-\left(\begin{array}{c}
2 n \\
k
\end{array}\right)\right) \frac{u_{n-k}(-1,1)}{n-k} \\
& =\frac{1}{n} \sum_{k=0}^{n-1}\left(\begin{array}{c}
2 n \\
k
\end{array}\right) u_{n-k}(-1,1)=\frac{1}{n} \sum_{k=0}^{n-1}\left(\begin{array}{c}
2 k \\
k
\end{array}\right)\left(\bmod 3^{\nu_{3}(m-1)-1}\right) .
\end{aligned}
$$

Thus (1.3) easily follows from (1.1).

Suppose that $a \geq \nu_{3}(m-1)$. When $m=4$, we have $u_{n}(2,1)=n$. By (2.5),

$$
\begin{aligned}
\frac{1}{3^{a}} \sum_{k=0}^{3^{a}-1} \frac{1}{4^{k}}\left(\begin{array}{c}
2 k \\
k
\end{array}\right) & =\frac{1}{3^{a} \cdot 4^{3^{a}-1}} \sum_{k=0}^{3^{a}-1}\left(\begin{array}{c}
2 \cdot 3^{a} \\
k
\end{array}\right)\left(3^{a}-k\right) \\
& =\frac{1}{4^{3^{a}-1}} \sum_{k=0}^{3^{a}-1}\left(\begin{array}{c}
2 \cdot 3^{a} \\
k
\end{array}\right)-\frac{2}{4^{3^{a}-1}} \sum_{k=1}^{3^{a}-1}\left(\begin{array}{c}
2 \cdot 3^{a}-1 \\
k-1
\end{array}\right) \\
& \equiv \sum_{k=0}^{3^{a}-1}\left(\begin{array}{c}
2 \cdot 3^{a} \\
k
\end{array}\right)+\sum_{k=1}^{3^{a}-1}\left(\begin{array}{c}
2 \cdot 3^{a}-1 \\
k-1
\end{array}\right) \equiv 1(\bmod 3) .
\end{aligned}
$$

Suppose that $m \neq 4$. Note that

$$
\begin{aligned}
& \sum_{k=0}^{3^{a}-1}\left(2\left(\begin{array}{c}
2 \cdot 3^{a}-1 \\
k
\end{array}\right)-\left(\begin{array}{c}
2 \cdot 3^{a} \\
k
\end{array}\right)\right) \cdot \frac{u_{3^{a}-k}(-1,1)}{3^{a}-k} \\
= & \frac{1}{3^{a}} \sum_{k=0}^{3^{a}-1}\left(\begin{array}{c}
2 \cdot 3^{a} \\
k
\end{array}\right) u_{3^{a}-k}(-1,1)=\frac{1}{3^{a}} \sum_{k=0}^{3^{a}-1}\left(\begin{array}{c}
2 k \\
k
\end{array}\right) \equiv 0\left(\bmod 3^{a}\right) .
\end{aligned}
$$

Hence applying (2.1) and (2.7), we get

$$
\begin{aligned}
\frac{1}{3^{a}} \sum_{k=0}^{3^{a}-1} \frac{1}{m^{k}}\left(\begin{array}{c}
2 k \\
k
\end{array}\right) & =\frac{1}{m^{3^{a}-1}} \sum_{k=0}^{3^{a}-1}\left(2\left(\begin{array}{c}
2 \cdot 3^{a}-1 \\
k
\end{array}\right)-\left(\begin{array}{c}
2 \cdot 3^{a} \\
k
\end{array}\right)\right) \cdot \frac{u_{3^{a}-k}(m-2,1)}{3^{a}-k} \\
& \equiv \sum_{k=0}^{3^{a}-1}\left(2\left(\begin{array}{c}
2 \cdot 3^{a}-1 \\
k
\end{array}\right)-\left(\begin{array}{c}
2 \cdot 3^{a} \\
k
\end{array}\right)\right) \cdot \frac{m-1}{3}\left(\begin{array}{c}
3^{a}-k-1 \\
2
\end{array}\right) \\
& \equiv \frac{m-1}{3}\left(\sum_{k=0}^{3^{a}-1} 2\left(\begin{array}{c}
2 \cdot 3^{a}-1 \\
k
\end{array}\right)\left(\begin{array}{c}
2 \cdot 3^{a}-k-1 \\
2
\end{array}\right)-\left(\begin{array}{c}
3^{a}-1 \\
2
\end{array}\right)\right) \\
& =\frac{m-1}{3}\left(\sum_{k=0}^{3^{a}-1}\left(2 \cdot 3^{a}-1\right)\left(2 \cdot 3^{a}-2\right)\left(\begin{array}{c}
2 \cdot 3^{a}-3 \\
k
\end{array}\right)-\left(\begin{array}{c}
3^{a}-1 \\
2
\end{array}\right)\right) \\
& \equiv \frac{m-1}{3}\left(\bmod 3^{\nu_{3}(m-1)}\right),
\end{aligned}
$$


where in the last step we use the fact

$$
\sum_{k=0}^{3^{a}-1}\left(\begin{array}{c}
2 \cdot 3^{a}-3 \\
k
\end{array}\right)=2^{2 \cdot 3^{a}-4}+\frac{1}{2}\left(\begin{array}{c}
2 \cdot 3^{a}-3 \\
3^{a}-2
\end{array}\right)+\left(\begin{array}{c}
2 \cdot 3^{a}-3 \\
3^{a}-1
\end{array}\right) \equiv 1(\bmod 3) .
$$

This proves (1.4).

\section{Proofs of (1.5) AND (1.7)}

In this section, we shall prove (1.5) and (1.6), under the assumption of the following congruence:

$$
\sum_{k=0}^{n-1}(-1)^{k}\left(\begin{array}{c}
n-1 \\
k
\end{array}\right)\left(\begin{array}{c}
2 k \\
k
\end{array}\right) \equiv 0\left(\bmod 3^{2 \nu_{3}(n)-1}\right) .
$$

And the proof of (3.1) will be given in the final section. We also need a special case of an identity of Sun [2, (2.6)]:

$$
\frac{1}{n} \sum_{k=0}^{n-1} \frac{(-1)^{k}}{m^{k}}\left(\begin{array}{c}
n-1 \\
k
\end{array}\right)\left(\begin{array}{c}
2 k \\
k
\end{array}\right)=\sum_{k=1}^{n} \frac{(-1)^{k-1}}{k}\left(\begin{array}{c}
n-1 \\
k-1
\end{array}\right) \sum_{l=0}^{k-1} \frac{1}{m^{l}}\left(\begin{array}{c}
2 l \\
l
\end{array}\right) .
$$

Thus from (2.3), (2.7) and (3.1), it follows that

$$
\begin{aligned}
& \frac{1}{n} \sum_{k=0}^{n-1} \frac{(-1)^{k}}{m^{k}}\left(\begin{array}{c}
n-1 \\
k
\end{array}\right)\left(\begin{array}{c}
2 k \\
k
\end{array}\right) \\
= & \sum_{k=1}^{n} \frac{(-1)^{k-1}}{m^{k-1}}\left(\begin{array}{c}
n-1 \\
k-1
\end{array}\right) \sum_{l=0}^{k-1}\left(2\left(\begin{array}{c}
2 k-1 \\
l
\end{array}\right)-\left(\begin{array}{c}
2 k \\
l
\end{array}\right)\right) \frac{u_{k-l}(m-2,1)}{k-l} \\
\equiv & \sum_{k=1}^{n} \frac{(-1)^{k-1}}{m^{k-1}}\left(\begin{array}{c}
n-1 \\
k-1
\end{array}\right) \sum_{l=0}^{k-1}\left(2\left(\begin{array}{c}
2 k-1 \\
l
\end{array}\right)-\left(\begin{array}{c}
2 k \\
l
\end{array}\right)\right) \frac{u_{k-l}(-1,1)}{k-l} \\
\equiv & \frac{1}{n} \sum_{k=0}^{n-1} \frac{(-1)^{k-1}}{m^{k-1}}\left(\begin{array}{c}
n-1 \\
k
\end{array}\right)\left(\begin{array}{c}
2 k \\
k
\end{array}\right) \equiv 0\left(\bmod 3^{\nu_{3}(m-1)-1}\right) .
\end{aligned}
$$

So (1.5) is concluded.

First, suppose that $m \neq 4$. For an integer $a \geq \nu_{3}(m-1)+1$, define

$$
f(a)=\sum_{k=1}^{3^{a}} \frac{(-1)^{k-1}}{m^{k-1}}\left(\begin{array}{c}
3^{a}-1 \\
k-1
\end{array}\right) \sum_{l=0}^{k-1}\left(2\left(\begin{array}{c}
2 k-1 \\
l
\end{array}\right)-\left(\begin{array}{c}
2 k \\
l
\end{array}\right)\right)\left(\begin{array}{c}
k-l-1 \\
2
\end{array}\right) .
$$


By (2.1),

$$
\begin{aligned}
& \sum_{k=1}^{3^{a}} \frac{(-1)^{k-1}}{m^{k-1}}\left(\begin{array}{c}
3^{a}-1 \\
k-1
\end{array}\right) \sum_{l=0}^{k-1}\left(2\left(\begin{array}{c}
2 k-1 \\
l
\end{array}\right)-\left(\begin{array}{c}
2 k \\
l
\end{array}\right)\right) \frac{u_{k-l}(m-2,1)}{k-l} \\
\equiv & \sum_{k=1}^{3^{a}} \frac{(-1)^{k-1}}{m^{k-1}}\left(\begin{array}{c}
3^{a}-1 \\
k-1
\end{array}\right) \sum_{l=0}^{k-1}\left(2\left(\begin{array}{c}
2 k-1 \\
l
\end{array}\right)-\left(\begin{array}{c}
2 k \\
l
\end{array}\right)\right) \frac{u_{k-l}(-1,1)}{k-l} \\
& +\sum_{k=1}^{3^{a}} \frac{(-1)^{k-1}}{m^{k-1}}\left(\begin{array}{c}
3^{a}-1 \\
k-1
\end{array}\right) \sum_{l=0}^{k-1}\left(2\left(\begin{array}{c}
2 k-1 \\
l
\end{array}\right)-\left(\begin{array}{c}
2 k \\
l
\end{array}\right)\right) \cdot \frac{m-1}{3}\left(\begin{array}{c}
k-l-1 \\
2
\end{array}\right) \\
= & \frac{1}{3^{a}} \sum_{k=0}^{3^{a}-1} \frac{(-1)^{k-1}}{m^{k-1}}\left(\begin{array}{c}
3^{a}-1 \\
k
\end{array}\right)\left(\begin{array}{c}
2 k \\
k
\end{array}\right)+\frac{m-1}{3} f(a)\left(\bmod 3^{\nu_{3}(m-1)}\right) .
\end{aligned}
$$

Thus in view of (3.1), it suffices to show that

$$
f(a) \equiv-1(\bmod 3) .
$$

Noting that

$$
\left(\begin{array}{c}
k-l-1 \\
2
\end{array}\right)=\frac{(k-l-1)(k-l-2)}{2} \equiv \begin{cases}1(\bmod 3), & \text { if } 3 \mid k-l, \\
0(\bmod 3), & \text { otherwise }\end{cases}
$$

we have

$$
\begin{aligned}
& \sum_{l=0}^{k-1}\left(2\left(\begin{array}{c}
2 k-1 \\
l
\end{array}\right)-\left(\begin{array}{c}
2 k \\
l
\end{array}\right)\right)\left(\begin{array}{c}
k-l-1 \\
2
\end{array}\right) \\
\equiv & \sum_{\substack{0 \leq j \leq k-l \\
3 \mid k-l}}\left(2\left(\begin{array}{c}
2 k-1 \\
l
\end{array}\right)-\left(\begin{array}{c}
2 k \\
l
\end{array}\right)\right)(\bmod 3) .
\end{aligned}
$$

By the proof of [2, Theorem 1.1],

$$
\sum_{\substack{0 \leq j \leq k-l \\
3 \mid k-l}}\left(2\left(\begin{array}{c}
2 k-1 \\
l
\end{array}\right)-\left(\begin{array}{c}
2 k \\
l
\end{array}\right)\right) \equiv\left(\begin{array}{c}
2 k / 3^{\nu_{3}(k)}-1 \\
k / 3^{\nu_{3}(k)}-1
\end{array}\right)(\bmod 3) .
$$

Apparently for $0 \leq k \leq 3^{a}-1$,

$$
\left(\begin{array}{c}
3^{a}-1 \\
k
\end{array}\right)=\prod_{j=1}^{k}\left(\frac{3^{a}}{j}-1\right) \equiv(-1)^{k}(\bmod 3) .
$$


Hence noting that $a \geq 2$ and applying (1.1), we can get

$$
\begin{aligned}
f(a) & \equiv \sum_{k=1}^{3^{a}}(-1)^{k-1}\left(\begin{array}{c}
3^{a}-1 \\
k-1
\end{array}\right)\left(\begin{array}{c}
2 k / 3^{\nu_{3}(k)}-1 \\
k / 3^{\nu_{3}(k)}-1
\end{array}\right) \equiv \sum_{j=1}^{a} \sum_{\substack{1 \leq i \leq 3^{a-j} \\
3 \nmid i}}\left(\begin{array}{c}
2 i-1 \\
i-1
\end{array}\right) \\
& =1+\frac{1}{2} \sum_{j=1}^{a-1}\left(\sum_{i=0}^{3^{a-j}-1}\left(\begin{array}{c}
2 i \\
i
\end{array}\right)-\sum_{i=0}^{3^{a-j-1}-1}\left(\begin{array}{c}
6 i \\
3 i
\end{array}\right)\right) \\
& \equiv 1+\frac{1}{2} \sum_{j=1}^{a-1}\left(\sum_{i=0}^{3^{a-j}-1}\left(\begin{array}{c}
2 i \\
i
\end{array}\right)-\sum_{i=0}^{3^{a-j-1}-1}\left(\begin{array}{c}
2 i \\
i
\end{array}\right)\right) \equiv 1-\frac{1}{2} \equiv-1(\bmod 3) .
\end{aligned}
$$

Finally, if $m=4$, then we have

$$
\begin{aligned}
\frac{1}{3^{a}} \sum_{k=0}^{3^{a}-1} \frac{(-1)^{k}}{4^{k}}\left(\begin{array}{c}
3^{a}-1 \\
k
\end{array}\right)\left(\begin{array}{c}
2 k \\
k
\end{array}\right) & =\sum_{k=1}^{3^{a}}\left(\begin{array}{c}
3^{a}-1 \\
k-1
\end{array}\right) \frac{(-1)^{k-1}}{k} \sum_{l=0}^{k-1} \frac{1}{4^{l}}\left(\begin{array}{c}
2 l \\
l
\end{array}\right) \\
& \equiv \sum_{k=1}^{3^{a}} \frac{1}{k} \sum_{l=0}^{k-1}\left(\begin{array}{c}
2 l \\
l
\end{array}\right)(\bmod 3) .
\end{aligned}
$$

Clearly,

$$
\sum_{l=0}^{k-1} \frac{1}{4^{l}}\left(\begin{array}{c}
2 l \\
l
\end{array}\right)=\sum_{l=0}^{k-1}(-1)^{l}\left(\begin{array}{c}
-1 / 2 \\
l
\end{array}\right)=(-1)^{k-1}\left(\begin{array}{c}
-1 / 2-1 \\
k-1
\end{array}\right)=\frac{k}{2^{2 k-1}}\left(\begin{array}{c}
2 k \\
k
\end{array}\right) .
$$

So using (1.1) again, we obtain that

$$
\frac{1}{3^{a}} \sum_{k=0}^{3^{a}-1} \frac{(-1)^{k}}{4^{k}}\left(\begin{array}{c}
3^{a}-1 \\
k
\end{array}\right)\left(\begin{array}{c}
2 k \\
k
\end{array}\right) \equiv 2 \sum_{k=1}^{3^{a}}\left(\begin{array}{c}
2 k \\
k
\end{array}\right) \equiv 2\left(\left(\begin{array}{c}
2 \cdot 3^{a} \\
3^{a}
\end{array}\right)-1\right) \equiv-1(\bmod 3) .
$$

4. Proofs of (1.7) AND (3.1)

The key of the proofs of (1.7) and (3.1) is the following identity.

Lemma 4.1.

$$
\sum_{k=0}^{n}\left(\begin{array}{c}
2 k \\
k
\end{array}\right)\left(\begin{array}{l}
n \\
k
\end{array}\right)(-x)^{k}=\frac{1}{4^{n}} \sum_{k=0}^{n}\left(\begin{array}{c}
2 j \\
j
\end{array}\right)\left(\begin{array}{c}
2(n-j) \\
n-j
\end{array}\right)(1-4 x)^{k} .
$$


Proof.

$$
\begin{aligned}
\sum_{k=0}^{n}\left(\begin{array}{c}
2 k \\
k
\end{array}\right)\left(\begin{array}{l}
n \\
k
\end{array}\right)(-x)^{k} & =\sum_{k=0}^{n}\left(\begin{array}{c}
-1 / 2 \\
k
\end{array}\right)\left(\begin{array}{l}
n \\
k
\end{array}\right)(4 x-1+1)^{k} \\
& =\sum_{k=0}^{n}\left(\begin{array}{c}
-1 / 2 \\
k
\end{array}\right)\left(\begin{array}{l}
n \\
k
\end{array}\right) \sum_{j=0}^{k}\left(\begin{array}{l}
k \\
j
\end{array}\right)(4 x-1)^{j} \\
& =\sum_{j=0}^{n}\left(\begin{array}{c}
-1 / 2 \\
j
\end{array}\right)(4 x-1)^{j} \sum_{k=j}^{n}\left(\begin{array}{c}
-1 / 2-j \\
k-j
\end{array}\right)\left(\begin{array}{c}
n \\
n-k
\end{array}\right) \\
& =\sum_{j=0}^{n}\left(\begin{array}{c}
-1 / 2 \\
j
\end{array}\right)\left(\begin{array}{c}
n-1 / 2-j \\
n-j
\end{array}\right)(4 x-1)^{j} \\
& =\frac{1}{4^{n}} \sum_{j=0}^{n}\left(\begin{array}{c}
2 j \\
j
\end{array}\right)\left(\begin{array}{c}
2(n-j) \\
n-j
\end{array}\right)(1-4 x)^{j} .
\end{aligned}
$$

Substituting $x=1$ in (4.1), we get

$$
\sum_{k=0}^{n-1}(-1)^{k}\left(\begin{array}{c}
2 k \\
k
\end{array}\right)\left(\begin{array}{c}
n-1 \\
k
\end{array}\right)=\frac{1}{4^{n-1}} \sum_{k=0}^{n-1}(-3)^{k}\left(\begin{array}{c}
2 k \\
k
\end{array}\right)\left(\begin{array}{c}
2(n-1-k) \\
n-1-k
\end{array}\right) .
$$

Let $a=\nu_{3}(n)$. Obviously,

$$
\sum_{k=0}^{n-1}(-3)^{k}\left(\begin{array}{c}
2 j \\
j
\end{array}\right)\left(\begin{array}{c}
2(n-1-k) \\
n-1-k
\end{array}\right) \equiv \sum_{k=0}^{2 a-2}(-3)^{k}\left(\begin{array}{c}
2 k \\
k
\end{array}\right)\left(\begin{array}{c}
2(n-1-k) \\
n-1-k
\end{array}\right)\left(\bmod 3^{2 a-1}\right) .
$$

When $a=1$, writing $n=3 b$ with $3 \nmid b$, we have

$$
\sum_{k=0}^{0}(-3)^{k}\left(\begin{array}{c}
2 k \\
k
\end{array}\right)\left(\begin{array}{c}
2(n-1-k) \\
n-1-k
\end{array}\right)=\left(\begin{array}{c}
6 b-2 \\
3 b-1
\end{array}\right)
$$

is divisible by 3 . Below we only consider $a \geq 2$. Noting that

$$
\left(\begin{array}{c}
2(n-1) \\
n-1
\end{array}\right) \frac{\left(\begin{array}{c}
n-1 \\
k
\end{array}\right)^{2}}{\left(\begin{array}{c}
2(n-1) \\
2 k
\end{array}\right)}=\left(\begin{array}{c}
2 k \\
k
\end{array}\right)\left(\begin{array}{c}
2(n-1-k) \\
n-1-k
\end{array}\right)
$$

we have

$$
\sum_{k=0}^{n-1}(-1)^{k}\left(\begin{array}{c}
2 k \\
k
\end{array}\right)\left(\begin{array}{c}
n-1 \\
k
\end{array}\right) \equiv \frac{1}{4^{n-1}}\left(\begin{array}{c}
2(n-1) \\
n-1
\end{array}\right) \sum_{k=0}^{2 a-2}(-3)^{k} \frac{\left(\begin{array}{c}
n-1 \\
k
\end{array}\right)^{2}}{\left(\begin{array}{c}
n-1) \\
2 k
\end{array}\right)}\left(\bmod 3^{2 a-1}\right) .
$$

Since

$$
\left(\begin{array}{c}
2(n-1) \\
n-1
\end{array}\right)=\frac{n}{2(2 n-1)}\left(\begin{array}{c}
2 n \\
n
\end{array}\right) \equiv 0\left(\bmod 3^{a}\right)
$$


it suffices to prove that

$$
\sum_{k=0}^{2 a-2}(-3)^{k} \frac{\left(\begin{array}{c}
n-1 \\
k
\end{array}\right)^{2}}{\left(\begin{array}{c}
n-1) \\
2 k
\end{array}\right)} \equiv 0\left(\bmod 3^{a-1}\right) .
$$

Clearly,

$$
\frac{\left(\begin{array}{c}
n-1 \\
k
\end{array}\right)^{2}}{\left(\begin{array}{c}
2(n-1) \\
2 k
\end{array}\right)}=\frac{1}{2 k+1} \cdot \frac{\prod_{j=1}^{k}(1-n / j)^{2}}{\prod_{j=2}^{2 k+1}(1-2 n / j)} .
$$

For $1 \leq k \leq 2 a-2$ and $2 \leq j \leq 2 k+1$, it is easy to check that

$$
\nu_{3}(j) \leq a-1
$$

and

$$
\nu_{3}((2 k+1) j) \leq k+1
$$

Hence for $2 \leq j \leq k$

$$
\frac{3^{k}(1-n / j)}{2 k+1}=\frac{3^{k}}{2 k+1}-\frac{3^{k} n}{(2 k+1) j} \equiv \frac{3^{k}}{2 k+1}\left(\bmod 3^{a-1}\right) .
$$

Similarly, for $1 \leq j \leq 2 k+1$, we also have

$$
\frac{3^{k}}{(2 k+1)(1-2 n / j)} \equiv \frac{3^{k}}{2 k+1}\left(\bmod 3^{a-1}\right),
$$

since

$$
\frac{1}{1-2 n / j}=1+\frac{2 n}{j}+\left(\frac{2 n}{j}\right)^{2}+\cdots
$$

over the rational 3-adic field $\mathbb{Q}_{3}$. Thus we get

$$
\sum_{k=0}^{2 a-2}(-3)^{k} \frac{\left(\begin{array}{c}
n-1 \\
k
\end{array}\right)^{2}}{\left(\begin{array}{c}
2(n-1) \\
2 k
\end{array}\right)} \equiv \sum_{k=0}^{2 a-2} \frac{(-3)^{k}}{2 k+1}\left(\bmod 3^{a-1}\right) .
$$

Note that for $k \geq 2 a-1$, we always have

$$
\frac{3^{k}}{2 k+1} \equiv 0\left(\bmod 3^{a-1}\right)
$$

Thus (3.1) immediately follows from the following lemma.

\section{Lemma 4.2.}

$$
\sum_{k=0}^{\infty} \frac{(-3)^{k}}{2 k+1}
$$

vanishes over $\mathbb{Q}_{3}$. 
Proof. Let $\mathbb{C}_{3}$ denote the completion of the algebraic closure of $\mathbb{Q}_{3}$. For any $x \in \mathbb{C}_{3}$ with the 3 -adic norm $|x|_{3}<1$, define the 3-adic logarithm function

$$
\log _{3}(1+x)=\sum_{n=1}^{\infty} \frac{(-1)^{n+1} x^{n}}{n}
$$

Clearly,

$$
\begin{aligned}
\sum_{k=0}^{\infty} \frac{(-3)^{k}}{2 k+1} & =\frac{1}{\sqrt{-3}} \sum_{k=0}^{\infty} \frac{(\sqrt{-3})^{2 k+1}}{2 k+1}=\frac{1}{2 \sqrt{-3}}\left(\sum_{k=1}^{\infty} \frac{(\sqrt{-3})^{k}}{k}-\sum_{k=1}^{\infty} \frac{(-\sqrt{-3})^{k}}{k}\right) \\
& =\frac{1}{2 \sqrt{-3}}\left(\log _{3}(1+\sqrt{-3})-\log _{3}(1-\sqrt{-3})\right)=\frac{1}{2 \sqrt{-3}} \log _{3}\left(\frac{\sqrt{-3}-1}{2}\right)
\end{aligned}
$$

Since $(\sqrt{-3}-1) / 2$ is a third root of unity, the lemma is concluded.

The proof of (1.7) is very similar, only requiring a few additional discussions. Now we have

$$
\sum_{k=0}^{3^{a}-1}(-1)^{k}\left(\begin{array}{c}
2 k \\
k
\end{array}\right)\left(\begin{array}{c}
3^{a}-1 \\
k
\end{array}\right) \equiv \frac{1}{4^{3^{a}-1}}\left(\begin{array}{c}
2\left(3^{a}-1\right) \\
3^{a}-1
\end{array}\right) \sum_{k=0}^{2 a-1}(-3)^{k} \frac{\left(\begin{array}{c}
3^{a}-1 \\
k
\end{array}\right)^{2}}{\left(\begin{array}{c}
2\left(3^{a}-1\right) \\
2 k
\end{array}\right)}\left(\bmod 3^{2 a}\right) .
$$

Since

$$
\frac{1}{3^{a}}\left(\begin{array}{c}
2\left(3^{a}-1\right) \\
3^{a}-1
\end{array}\right)=\frac{1}{2\left(2 \cdot 3^{a}-1\right)}\left(\begin{array}{c}
2 \cdot 3^{a} \\
3^{a}
\end{array}\right) \equiv-1(\bmod 3)
$$

we only need to show that

$$
\sum_{k=0}^{2 a-1}(-3)^{k} \frac{\left(\begin{array}{c}
3^{a}-1 \\
k
\end{array}\right)^{2}}{\left(\begin{array}{c}
2\left(3^{a}-1\right) \\
2 k
\end{array}\right)} \equiv 3^{a-1}\left(\bmod 3^{a}\right)
$$

Since $a \geq 2$, for $1 \leq k \leq 2 a-1$ and $1 \leq j \leq 2 k+1$, we always have $\nu_{3}(j) \leq a-1$, and we also have $\nu_{3}((2 k+1) j) \leq k$ unless $k=1$ and $j=3$. Hence for $(k, j) \neq(1,3)$,

$$
\frac{3^{k}(1-n / j)}{2 k+1} \equiv \frac{3^{k}}{2 k+1}\left(\bmod 3^{a}\right) \quad \text { and } \quad \frac{3^{k}}{(2 k+1)(1-2 n / j)} \equiv \frac{3^{k}}{2 k+1}\left(\bmod 3^{a}\right) .
$$

That is, for $k \geq 2$,

$$
\frac{\left(\begin{array}{c}
3^{a}-1 \\
k
\end{array}\right)^{2}}{\left(\begin{array}{c}
2\left(3^{a}-1\right) \\
2 k
\end{array}\right)}=\frac{1}{2 k+1} \cdot \frac{\prod_{j=1}^{k}(1-n / j)^{2}}{\prod_{j=2}^{2 k+1}(1-2 n / j)} \equiv \frac{(-3)^{k}}{2 k+1}\left(\bmod 3^{a}\right) .
$$


It follows that

$$
\begin{aligned}
& \sum_{k=0}^{2 a-1}(-3)^{k} \frac{\left(\begin{array}{c}
3^{a}-1 \\
k
\end{array}\right)^{2}}{\left(\begin{array}{c}
2\left(3^{a}-1\right) \\
2 k
\end{array}\right)} \equiv \sum_{k=0}^{1}(-3)^{k} \frac{\left(\begin{array}{c}
3^{a}-1 \\
k
\end{array}\right)^{2}}{\left(\begin{array}{c}
2\left(3^{a}-1\right) \\
2 k
\end{array}\right)}+\sum_{k=2}^{2 a-1} \frac{(-3)^{k}}{2 k+1} \\
\equiv & 1+(-3) \cdot \frac{\left(\begin{array}{c}
3^{a}-1 \\
1
\end{array}\right)^{2}}{\left(\begin{array}{c}
2\left(3^{a}-1\right) \\
2
\end{array}\right)}=1-\frac{6\left(3^{a}-1\right)^{2}}{\left(2 \cdot 3^{a}-2\right)\left(2 \cdot 3^{a}-3\right)} \\
= & \frac{3^{a-1}}{1-2 \cdot 3^{a-1}} \equiv 3^{a-1}\left(\bmod 3^{a}\right) .
\end{aligned}
$$

Acknowledgment. We are grateful to Professor Zhi-Wei Sun for his helpful discussions on this paper.

\section{REFERENCES}

[1] M. Strauss, J. Shallit and D. Zagier, Some strange 3-adic identities, Amer. Math. Monthly, 99(1992), 66-69.

[2] Z. W. Sun, p-adic valuations of some sums of multinomial coefficients, Acta Arith., 148(2011), 63-76.

[3] Z. W. Sun and R. Tauraso, New congruences for central binomial coefficients, Adv. in Appl. Math., 45(2010), 125-148.

E-mail address: yongzhang1982@163.com

Department of Basic Course, Nanjing Institute of Technology, Nanjing 211167, People's Republic of China

E-mail address: haopan79@yahoo.com.cn

Department of Mathematics, Nanjing University, Nanjing 210093, People's RePUBLIC OF CHINA 